\title{
Factors that determine the adherence to treatment by patients with psoriasis - pilot study
}

\author{
${ }^{1 *}$ Grochowiec Marcelina, ${ }^{2}$ Lesiak Aleksandra, ${ }^{2}$ Narbutt Joanna and ${ }^{2}$ Bednarski Igor
}

${ }^{1}$ Dermatology and Venereology Department, Specialist Hospital in Jaslo, Lwowska St. 22, 38-200 Jaslo, Poland. ${ }^{2}$ Department of Dermatology, Pediatric Dermatology and Oncology Clinic, Kniaziewicza St. 1/5, 91 347 Lodz, Poland

*Corresponding Author's Email: m.grochowiec@wp.pl

\begin{abstract}
The level of adherence to treatment by patients with psoriasis is low. Previous studies showed that results of psoriasis treatment are significantly influenced by the level of medication adherence. We evaluated the level of adherence to treatment by patients with psoriasis and its association with Psoriasis Area and Severity Index (PASI), Body Surface Area (BSA), Dermatology Quality of Life Index (DLQI) using author's questionnaire.

The research included 60 patients with psoriasis. Skin lesions have been evaluated using PASI and BSA. Patients completed DLQI and the author's own questionnaire about adherence to treatment.

PASI $>10$ and $B S A>10$ was received by $66.6 \%$ of patients, $P A S I<10$ and $B S A<10-25 \%$ of patients. As much as $76.6 \%$ of patients declared that the disease moderately or significantly lowers their quality of life. According to the questionnaire, $41.7 \%$ patients suffering from psoriasis showed poor adherence to treatment. Patients with PASI>10 and BSA>10 and DLQI>6 achieved a statistically significant higher result (worse adherence) in the author's own questionnaire when compared to patients with $\mathrm{PASI}<10$ and $\mathrm{BSA}<10$ and $\mathrm{DLQI} \leq 6$.
\end{abstract}

A better understanding of the determinants of adherence can improve the outcomes of psoriasis treatment and lead to higher patient satisfaction.

Keywords: Psoriasis; Medication adherence; Psoriasis area and severity index; Body surface area; Dermatology quality of life index.

\section{INTRODUCTION}

Psoriasis as a chronic systemic disease is a major health and social-economic problem. In addition to treating the skin, patients affected with psoriasis are required to undergo the treatment of comorbidities such as arthritis, depression, cardiovascular, metabolic and autoimmune diseases. Concomitant diseases develop in patients with psoriasis statistically earlier than in general population, and thus require early introduction of diagnostics and specialized treatment, which, one the one hand, deteriorates the quality of patients' personal and social life, and on the other hand, generates expenses for the medical care system (Oliveira et al., 2015). For that reason, factors that would enhance the effectiveness of psoriasis treatment are still sought. A few studies carried out so far highlight the issue of the adherence to treatment in chronic 
diseases such as psoriasis. The adherence to treatment is generally defined as the extent to which the patient's behaviour in treating the disease is consistent with recommendations provided by a physician. It was figured out that the higher the level of the adherence to treatment, the better treatment outcome (Zschocke et al., 2014; Rycombel et al., 2014). However, the research results demonstrate a low level of the adherence to treatment by patients suffering from psoriasis and low awareness of the health care system about significance of this problem (Nelson et al., 2013). The World Health Organization (WHO) has acknowledged the adherence to treatment in diseases such as psoriasis as one of the most essential factors contributing to success of the therapy (World Health Organization, 2003). Objective measurement of the adherence to treatment is difficult as it is conditioned by several factors concerning a patient, including approach to disease, social-economic status, psychological profile as well as factors associated with the therapy such as drug dosage method, occurrence of side effects, drug type, therapy expenses, efficiency and the health care system, including easy access to medical specialist and proper doctor-patient communication. There are available various models available to assess the adherence to treatment, but their drawback is the lack of approach directed towards dermatological patients whose specific course of diseases is significantly different from other chronic disorders (Arnet et al., 2015). Therefore, we consider it important to develop strategies aimed at improving the adherence to treatment by patients suffering from psoriasis as this will contribute to better treatment results, prevent frequent exacerbations of psoriasis, enable better control of comorbidities with dermatosis and improve the overall quality of life of patients with psoriasis. In this article, we will assess the level of the adherence to treatment and review the causes of non-adherence to treatment by patients suffering from psoriasis.

\section{OBJECTIVES}

To estimate the level of the adherence to treatment by patients suffering from psoriasis based on the author's questionnaire and its association with Psoriasis Area and Severity Index (PASI), Body Surface Area (BSA) and Dermatology Quality of Life Index (DLQI). The survey served to identify factors that impair the adherence to treatment by patients with psoriasis included in the study group.

\section{MATERIALS AND METHODS}

The conducted study is a pilot study and takes into account patients with plaque psoriasis. It has been carried out by dermatologists. The study included 60 patients hospitalized in the Department of Dermatology and Venereology of the Specialist Hospital in Jasło and those under care in the hospital outpatient dermatological clinic in 2015-2017. The criteria for including in the group was age $\geq 18$, diagnosed plaque psoriasis and no systemic treatment of psoriasis for at least 6 months prior to inclusion in the study. Skin lesions were evaluated using PASI-psoriasis severity index and BSA-percentage index of body surface area affected by psoriatic lesions. According to the Polish Dermatological Society, PASI $>10$ and $\mathrm{BSA}>10$ determines moderate and severe psoriasis, whereas PASI $\leq 10$ and $\mathrm{BSA} \leq 10$ mild character of the disease (Jevtić T et al., 2013). Patients also completed DLQI that consisted of 10 questions referring to different areas of patients' lives who suffer from dermatological diseases. Interpretation of DLQI results: 01 no impact of the disease on the quality of life, 2-5-low impact, 10-moderate impact, 11-20-very high impact and 21-30-extremely high impact (Szepietowski et al., 2014).

Patients filled in an author's questionnaire comprising 13 questions concerning the adherence to treatment by dermatological patients (Table 1). The questionnaire was anonymous to increase the probability of objective answers to questions. Questions used in the questionnaire were prepared dependent on the current knowledge on the factors determining the course of psoriasis, based on original studies and systematic reviews. The content of questions in the questionnaire concerned the following issues: regularity in the use of prescribed drugs, the impact of media and unconventional medicine, the influence of consistency and stains left by drugs on clothing, mental state of patients associated with the daily necessity of taking drugs, the impact of medical care on the treatment. The results of questionnaires were compared using statistical calculations with the results of PASI, BSA and DLQI. An analysis of answers to given questions was also carried out to identify the most common factors disrupting the adherence to treatment in the study group. Statistical data in the conducted study were collected using computer software STATISTICA 12.0 (StatSoft, Poland). The study was approved by the Bioethics Committee of Medical University of Lodz (No. RNN/113/16/KE). 
Table 1: Author's questionnaire.

Dear Patient!

Do you happen to

1. Forget to take drugs or apply prescribed ointment/cream? YES SOMETIMES NO

2. Stop or do not take drugs because of various information given on TV/radio/ Internet? YES SOMETIMES NO

3. Stop taking your drugs and start taking herbal preparations, home treatment or homeopathic preparations?

YES SOMETIMES NO

4. Forget to go to the pharmacy to buy prescribed drug?

YES SOMETIMES NO

5. Forget to take all your drugs on a journey?

YES SOMETIMES NO

6. Discontinue the treatment when the skin condition has improved?

YES SOMETIMES NO

7. Stop applying ointment/cream due to its bad consistency, smell or dirty clothes? YES SOMETIMES NO

8. Skip doses of prescribed drugs or another ointment/cream applications? YES SOMETIMES NO

9. Feel fatigue and mental burden due to daily obligation to take drugs? YES SOMETIMES NO

10. Need a second person to remind you about taking prescribed drugs or applying ointment/cream? YES SOMETIMES NO

11. Be not informed sufficiently by your doctor/nurse about drug dosage and its application method? YES SOMETIMES NO

12. Forget what your doctor said in the office?

YES SOMETIMES NO

13. Skip a check-up visit?

YES SOMETIMES NO

Yes-1 point, Sometimes-0.5 point, No-0 points.

0-1.5 points-good adherence to treatment

$>1.5$ points-poor adherence to treatment

\section{RESULTS}

The study was completed by all patients. The result PASI $>10$ and BSA $>10$ was achieved by 40 patients $(66.6 \%)$, while PASI $\leq 10$ and BSA $\leq 10$ was obtained by 15 patients $(25 \%)$. The DLQI results in the study group ranged from 2 to 20 points. 46 patients (76.6\%) received the DLQI result $\geq 6$ and 14 of them (23.4\%) had the DLQI result $<6$. The author's questionnaire included 35 patients $(58 \%)$ with a score of $\leq 1.5$, while 25 patients (42\%) achieved a result of $>1.5$. Patients with PASI $>10$ and BSA $>10$ obtained statistically higher result in the author's questionnaire when compared to patients with PASI $\leq 10$ and BSA $\leq 10$ (Figures 1 and 2). 


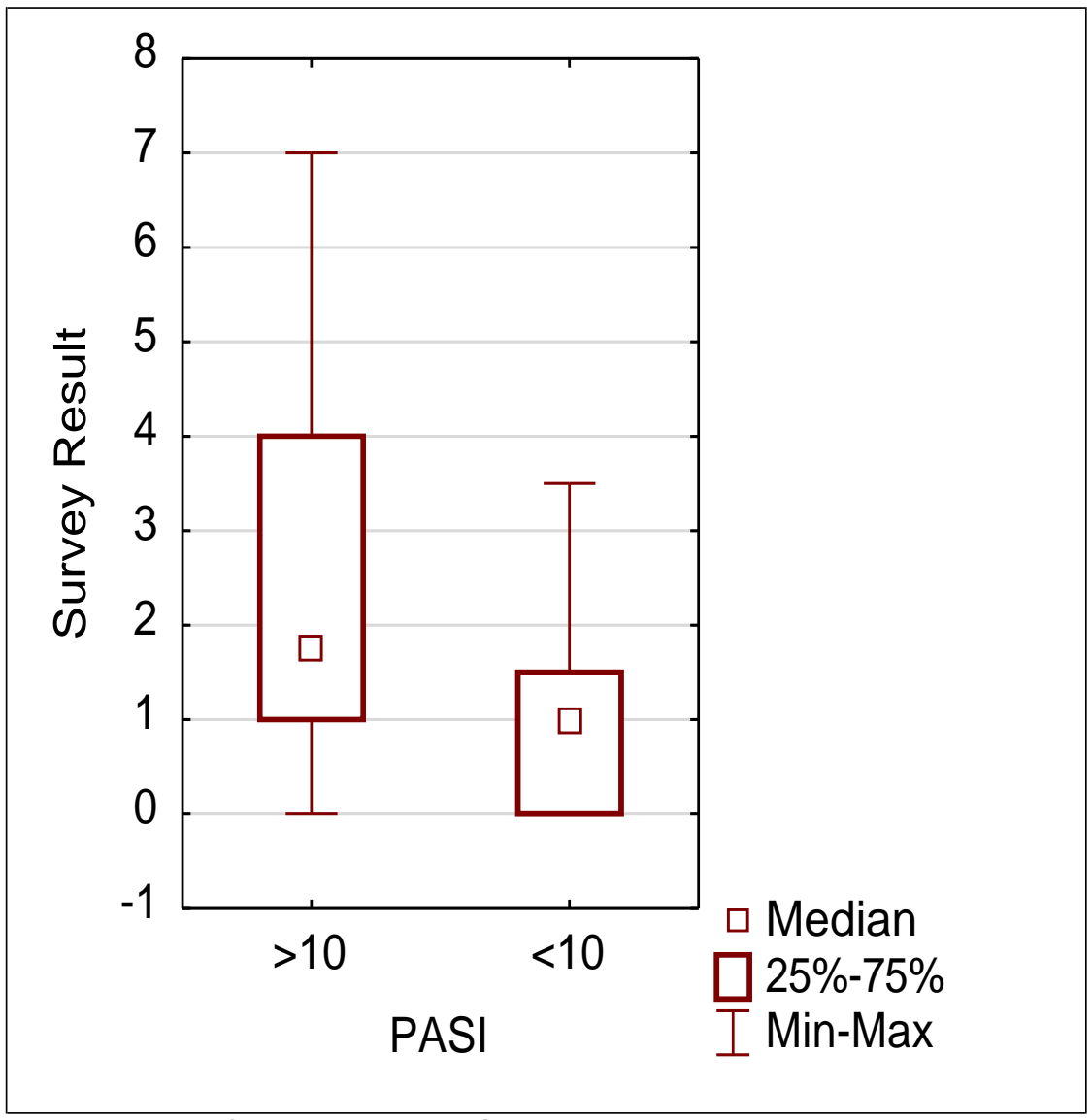

Figure 1: Comparison of PASI values to the questionnaire results.

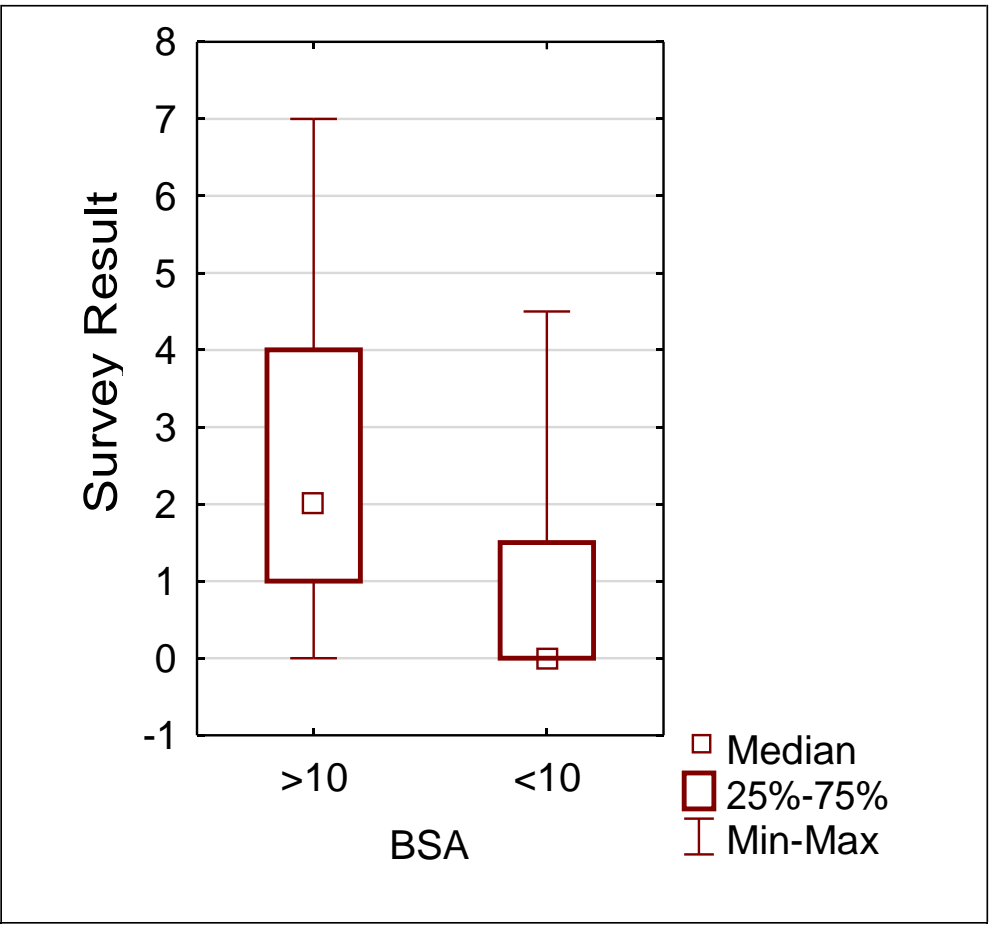

Figure 2: Comparison of BSA values to the questionnaire results. 
Patients with DLQI>6 demonstrated statistically considerably higher result in the author's questionnaire than those with DLQI $\leq 6$ (Figure 3).

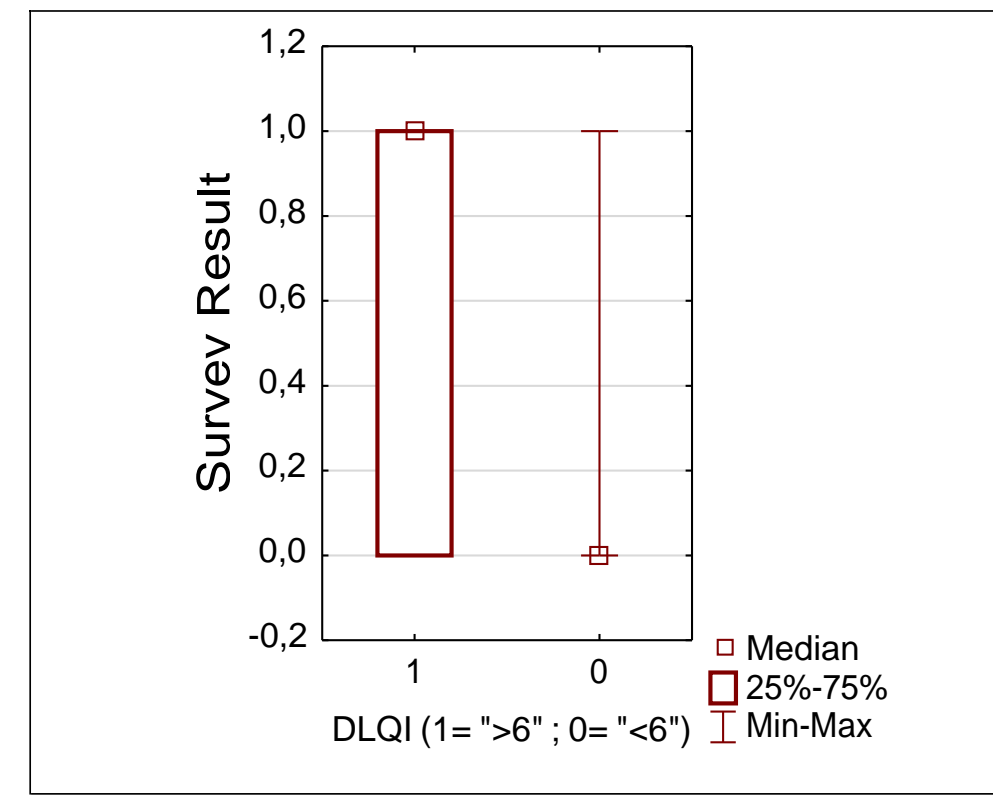

Figure 3: Comparison of $D L Q|>6 / D L Q| \leq 6$ values to the questionnaire results.

Patients from the study group answered 13 questions contained in the author's questionnaire. The distribution of their answers is presented in Figure 4. The most frequent cause of poor adherence to treatment was the feeling of fatigue and mental burden due to daily obligation to take drugs and forgetting about the necessity of using the drugs.

\section{Distribution of answers to questions}

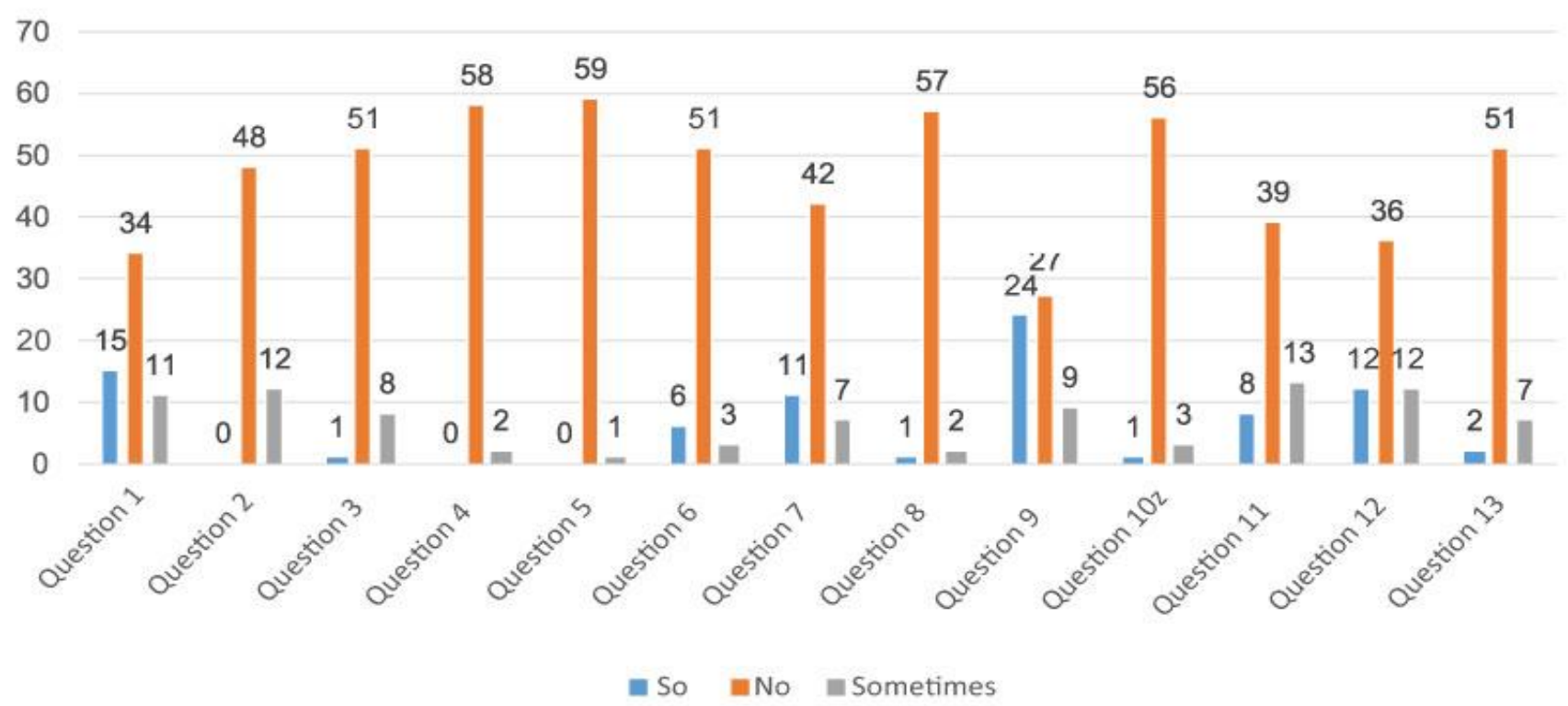

Figure 4: Distribution of answers to questions - whole group.

\section{DISCUSSION}

Psoriasis is a common skin diseases that occur in up to $1-3 \%$ of the general population. Few conducted studies point out a low level of the adherence to treatment by patients suffering from psoriasis, which leads to a lack of control over the development of this disease and poor treatment results. Our study demonstrated that as many as $42 \%$ of patients suffering from psoriasis showed poor adherence to treatment. There is little research of this type available in the literature, but it also indicates that about $40 \%$ of patients with psoriasis do not adhere to treatment (Jevtić et al., 2013; Choi et al., 2017). The systematic 
review by Augustine et al., 2011, based on 35 original publications demonstrates that the degree of the compliance with therapeutic recommendations by patients suffering from psoriasis ranged from $27 \%$ to $97 \%$ and depended largely on the type of the prescribed drug and the support provided by the health care system (Augustin et al., 2011). According to our study, the statistically higher severity of skin lesions expressed in PASI and BSA, the lower level of the adherence to treatment. This observation is confirmed by the study carried out by Zaghloul et al., who took into consideration 201 patients with psoriasis and indicated that the adherence to treatment was significantly lower in patients with higher disease severity and lesions localized on the face (Zaghloul et al., 2004). Jevtić et al., observed a considerable improvement of PASI in patients who started to follow treatment schemes (Jevtić et al., 2013). In turn, Thorneloe et al., showed similarly low level of the adherence to treatment by patients irrespective of the treatment method and the severity of psoriasis (Thorneloe et al., 2013).

Psoriasis as a chronic skin disease has a large impact on the patient's mental state and social relationships. A sense of stigma, isolation, fear of rejection by a 'healthy' part of the society may lead to psychological disorders such as depression, anxiety disorders or addictions. Such an impact on everyday life of people suffering from chronic skin diseases resulted in the DLQI questionnaire, which became a standard test for patients with psoriasis to assess their quality of life (Basińska et al., 2013). The conducted study shows that over $76 \%$ of patients indicated that moderate or severe psoriasis deteriorated their quality of life (expressed in DLQI), which significantly statistically correlated with worse adherence to treatment. There are very few studies describing this relationship available in the literature. Zaghloul et al., demonstrated inversely proportional relationship between DLQI and the adherence to treatment in patients suffering from psoriasis (Zaghloul et al., 2004). On the other hand, Bewley et al., point out that one of the main factors behind poor adherence to treatment by patients with psoriasis are depressive disorders, suicidal thoughts and sexual problems (Bewley et al., 2011).

According to our study, the most frequent cause of poor adherence to treatment included a feeling of mental burden caused by everyday obligation to take drugs and forgetting to take prescribed drugs. This is confirmed by other studies which indicate that the requirement to take drugs everyday (concerning mostly topical medications) and too time-consuming treatment are the main factors reducing the adherence to treatment (Piaserico et al., 2017). This observation is confirmed by research on patients with psoriasis taking biologics, which points out nearly $100 \%$ adherence to treatment regimens. This is due to no necessity of taking biological medicines every day and their quick results (Chan et al., 2013). According to Blome et al., time that the patient has to sacrifice for the treatment of the disease is the primary predictor of the loss of quality of life in psoriasis and poor adherence to treatment (Blome et al. 2010). Another important fact is information provided by the medical staff to patients on preferences regarding a medicine, frequency and duration of its application or use. Even when lesions are found to have disappeared, advising patients to continue psoriasis treatment is required in order to control subclinical inflammation and prevent relapse. Physicians should also schedule more frequent check-ups to improve the adherence to treatment. Each patient should be treated individually. Studies demonstrate that well-informed patients about characteristics of the disease, effectiveness, side effects and duration of the therapy achieve statistically higher level of the adherence to treatment (Chan et al., 2013; Yélamos et al., 2015).

\section{LIMITATIONS AND ADVANTAGES OF THE STUDY}

The limitation of the conducted study is a small number of patients in terms of the problem that is the adherence to treatment, which applies to every patient with psoriasis. The questionnaire was not validated. Our study indicates the fact that not only research on new medicines for psoriasis, or research on the immunology and genetics of this disease lead to improved treatment efficiency, but also simple methods such as providing patients with practical advice and improving communication between health service and patients may turn out to be more effective.

\section{CONCLUSIONS}

Evaluation of the adherence to treatment is of great importance in optimal therapy. The problem is to spread this kind of approach among physicians and the selection of the appropriate measurement method. Supporting research on the causes of the failure to adhere to treatment by patients suffering from psoriasis will lead to greater effectiveness of the treatment process.

\section{CONFLICT OF INTERESTS}

The authors declare no conflict of interests. 


\section{REFERENCES}

Arnet I, Metaxas C, Walter PN, Morisky DE, Hersberger KE (2015). The 8-item Morisky Medication Adherence Scale translated in German and validated against objective and subjective polypharmacy adherence measures in cardiovascular patients. Clin Prac 21(2), 271-77.

Augustin M, Holland B, Dartsch D, Langenbruch A, Radtke MA (2011). Adherence in the treatment of psoriasis: a systematic review. Dermatology 222(4), 363-374.

Basińska M, Szymańska L (2013). Związek nastroju z cechami choroby u osób z łuszczycą. Przegl Dermatol 100(3), 146-153.

Bewley A, Page BJ (2011). Maximizing patient adherence for optimal outcomes in psoriasis. Eur Acad Dermatol Venereol 25(4), 9-14.

Blome C, Simianer S, Purwins S, Laass A, Rustenbach SJ, Schaefer I, Radtke M, Augustin M (2010). Time needed for treatment is the major predictor of quality of life in psoriasis. Dermatology 221(2), 154-159.

Chan SA, Hussain F, Lawson LG, Ormerod AD (2013). Factors affecting adherence to treatment of psoriasis: comparing biologic therapy to other modalities. J Dermatolog Treat 24(1), 64-69.

Choi JW, Kim BR, Youn SW (2017). Adherence to Topical Therapies for the Treatment of Psoriasis: Surveys of Physicians and Patients. Ann Dermatol 29(5), 559-64.

Jevtić T, Bukumirić Z, Janković SM (2013). Effects of treatment adherence on clinical and economic outcomes in patients with psoriasis. Med Glas 10(1): 106-12.

Nelson P, Chew-Graham C, Griffiths C, Cordingley L (2013). Recognition of need in health care consultations: a qualitative study of people with psoriasis. Br J Dermatol 168(2), 354-361.

Oliveira Mde F, Rocha Bde O, Duarte GV (2015). Psoriasis: classical and emerging comorbidities. An Bras Dermatol 90(1), 9-20.

Piaserico S, Manfredini S, Borghi A, Gisondi P, Pazzaglia M, Stinco G, Venturini M, Conti A (2017). How to improve adherence to treatment in patients with mild-to-moderate psoriasis? An expert opinion. $G$ Ital Dermatol Venereol, 97-98.

Rycombel A, Lomper K, Uchmanowicz I (2014). Adherence i compliance w leczeniu nadciśnienia tętniczego. Arterial Hypertens 18(3), 151-158.

Szepietowski J, Adamski Z, Chodorowska G, Kaszuba A, Placek W, Rudnicka L, Reich A (2014). Leczenie łuszczycy-rekomendacje ekspertów Polskiego Towarzystwa Dermatologicznego. Część II: łuszczyca umiarkowana do ciężkiej. Przeg Dermatol 97(6), 1-13.

Thorneloe RJ, Bundy C, Griffiths CE, Ashcroft DM, Cordingley L (2013). Adherence to medication in patients with psoriasis: a systematic literature review. Br J Dermatol 168(1), 20-31.

World Health Organization: Adherence to long term therapy: evidence for action. Geneva: World Health Organization; 2003.

Yélamos O, Ros S, Puig L (2015). Improving patient outcomes in psoriasis: strategies to ensure treatment adherence. Psoriasis 17(5), 109-115.

Zaghloul SS, Goodfield MJD (2004). Objective assessment of compliance with psoriasis treatment. Arch Dermatol 140(4), 408-414.

Zschocke I, Mrowietz U, Karakasili E, Reich K (2014). Non-adherence and measures to improve adherence in the topical treatment of psoriasis. J EurAcad Dermatol Venereol 28(2), 4-9. 